\title{
DESCRIPCIÓN DE UNA ESPECIE NUEVA DE MELOE LINNAEUS, 1758 DEL SUBGÉNERO EURYMELOE REITTER, 1911 (COLEOPTERA, MELOIDAE) DEL NORTE DE MARRUECOS
}

\author{
J. L. Ruiz* \& M. García-París**
}

\begin{abstract}
RESUMEN
Se describe una especie nueva de Meloe (Coleoptera: Meloidae) del subgénero Eurymeloe, del grupo de M. rugosus, relacionada con especies del subgrupo de M. murinus. Los ejemplares estudiados de la especie nueva proceden del Rif occidental (Parque Nacional de Talassemtane, dorsal caliza rifeña), en el noroeste de Marruecos y se localizaron en hábitat forestal hiperhúmedo de montaña. La nueva especie se caracteriza por presentar: coloración negro mate con tonalidad grisácea; pilosidad blanquecino-amarillenta, muy corta, curvada y poco densa que no forma mechones; pronoto transverso, subrectangular, con surco medio longitudinal y depresiones longitudinales a ambos lados; punteado de cabeza y pronoto impreso y subconfluente; élitros apenas rugosos; artejos tarsales con cepillos pilosos poco desarrollados; edeago con lóbulos paramerales curvados con depresiones laterales y terminados en punta aguda, y lóbulo medio con el diente distal de la zona ventral cercano al ápice. El nuevo taxon es fenéticamente afín a $M$. nanus, M. baudueri y M. flavicomus, pero diferenciado de ellos tanto por los caracteres morfológicos externos como del edeago indicados. Esta especie, conocida únicamente de la localidad tipo, comparte área geográfica general con M. murinus, M. nanus y posiblemente M. baudueri, si bien hasta el momento no se ha detectado simpatría estricta con ninguna de ellas. El tipo de hábitat en el que se localiza la especie nueva, áreas de montaña con formaciones forestales mixtas de Pinus pinaster hamiltonii var. maghrebiana, P. nigra mauritanica y Abies maroccana (exclusivas del Rif calizo noroccidental), contrasta con los señalados para esas otras especies de Eurymeloe del subgrupo de M. (E.) murinus.
\end{abstract}

Palabras-clave: Taxonomía, Morfología, Coleoptera, Meloidae, Meloe, África, Marruecos, Faunística.

\section{ABSTRACT \\ Description of a new species of Meloe Linnaeus, 1758, subgenus Eurymeloe Reitter, 1911 (Coleoptera, Meloidae), from northern Morocco}

We describe a new species of Meloe (Coleoptera: Meloidae), subgenus Eurymeloe, included in the M. rugosus species group, related to species of the M. murinus subgroup. The specimens of the new species studied were found along the western Rif mountain chain (Talassemtane National Park) in northwestern Morocco, and were located in hiperhumid montane forest habitats. The new species is characterized by having: a dull black

* Instituto de Estudios Ceutíes. Paseo del Revellín 30. Apartado 593. 51080 Ceuta. España. euserica@hotmail.com

** Museo Nacional de Ciencias Naturales. C.S.I.C. c/ José Gutiérrez Abascal, 2. 28006 Madrid. España. mparis@mncn.csic.es 
coloration with greyish hue; a very short, curved, and not dense, yellow-whitish pilosity, which does not form tufts; a subrectangular transverse pronotum with a marked median grove and two longitudinal lateral depressions; head and pronotum punctures well impressed and subconfluent; elytra sligtly rugose; tarsomeres with slightly developed ventral pads; aedeagus with curved parameres, laterally depressed, apically pointed, with the distal ventral hook of median lobe close to apex. The new taxon es phenetically similar to M. nanus, M. baudueri and M. flavicomus, but distinguished from all of them by the mentioned morphological external and male genitalia characters. The distribution of the new species, only known from its type locality, overlaps those of M. murinus, $M$. nanus and possibly M. baudueri, but so far it has not been found in strict sympatry with any of them. The habitat type inhabited by the new species, mountain areas with mixed forests of Pinus pinaster hamiltonii var. maghrebiana, P. nigra mauritanica and Abies maroccana (endemic taxa of the limestone northwestern Rif mountain chain), contrasts with other habitat types recorded for species of the $M$. (E). murinus species subgroup of the subgenus Eurymeloe.

Key-words: Taxonomy, Morphology, Coleoptera, Meloidae, Meloe, Africa, Morocco, Faunistics.

\section{Introducción}

El subgénero Eurymeloe Reitter, 1911 [especietipo: Meloe brevicollis Panzer, 1793, por designación posterior (Pinto \& Selander, 1970)] agrupa en torno a 49 especies, aunque el estatus taxonómico de algunas es en la actualidad incierto (e.g. Bologna, 1988, 1990, 1991, 2008a), y junto al subgénero nominotípico constituyen los de mayor representación específica en el ámbito del género Meloe Linnaeus, 1758. Este hecho, unido a la homogeneidad morfológica del mismo (Bologna, 1988, 1991; Bologna et al., 1989) y a la escasez de material disponible para estudio, hacen que su taxonomía interna sea realmente compleja. La distribución geográfica del subgénero es fundamentalmente paleártica (incluidas las Islas Canarias y Madeira), si bien presenta una especie en las Islas Aleutianas, Meloe aleuticus Borchmann, 1942, en la zona de transición entre las regiones neártica y paleártica, una en Somalia, Meloe vignai Bologna, 1990, y otra en el Punjab (Paquistán), Meloe punjabensis Kaszab, 1958. La mayor diversidad taxonómica se centra en el ámbito circunmediterráneo y Asia media (Pinto \& Selander, 1970; Bologna, 1988, 1990, 1991, 2008a; Bologna et al., 1989).

La posición taxonómica de Eurymeloe ha sido objeto de debate en los últimos años. Así, Selander (1985) lo elevó a la categoría genérica, independiente de Meloe, y consideró sinónimos a los subgéneros Coelomeloe Reitter, 1911 (especie-tipo: Meloe tuccius Rossi, 1792, por monotipia) y Meloegonius Reitter, 1911 [especie-tipo: Meloe cicatricosus Leach, 1815, por designación posterior (Aksentjev, 1988)], criterio que mantiene posterior- mente para el primero de estos subgéneros (Selander, 1991) pero no para el segundo (Selander, 1989, 1991). Sin embargo, Bologna et al. (1989), Bologna \& Pinto $(1992,1995,1998)$ y Di Giulio et al. (2002), a partir del estudio comparado de las larvas de estadio I, concluyen que Eurymeloe ha de considerarse un mero subgénero de Meloe; hipótesis que apoyan Bologna \& Pinto (2001) mediante análisis filogenéticos basados en caracteres tanto larvarios como imaginales y comportamentales. Recientemente, Bologna \& Pinto (2002), Lückmann \& Scharf (2004) y Bologna (2008a) mantienen esta categoría subgenérica. No obstante, en lo concerniente a la sinonimia propuesta por Selander (1985, 1991) para Coelomeloe, Bologna (1991, 2008b), Bologna et al. (1989), Bologna \& Pinto (1995, 2002) y Di Giulio et al. (2002) señalan que posiblemente sea correcta, apoyada en la morfología larvaria muy similar, pero debido a las marcadas diferencias en la fase adulta estiman más adecuado mantenerlo temporalmente como subgénero válido, aunque filogenéticamente cercano a Eurymeloe.

Según Bologna (1988) y Bologna et al. (1989) Eurymeloe, a pesar de su elevada diversidad específica, se encuentra bien caracterizado en el seno de Meloe por presentar una morfología imaginal homogénea, larva de estadio I primitiva (tipo I de Selander, 1985), larva coarctata o hipnoteca de tegumentos blandos y, por último, comportamiento de cortejo sexual simple.

En cuanto a la taxonomía interna de este complejo subgénero, Bologna (1988), basándose fundamentalmente en la semejanza fenética (morfología imaginal), propone dos grupos de especies bien diferenciados, pero cuyas relaciones filogenéticas están 
aún por determinar: grupo I o grupo de Meloe rugosus Marsham, 1802, más homogéneo, que integra unas 25 especies caracterizadas por su color negro, tamaño medio-pequeño, pilosidad negra o amarillocastaña, punteado y rugosidad habitualmente marcadas, y antenas, en la mayoría de las especies, con artejos generalmente alargados y subcilíndricos, de lados paralelos; grupo II o grupo de Meloe brevicollis Panzer, 1793, que agrupa en torno a otras 24 especies (Bologna, 1988, 1991, y teniendo en cuenta la más reciente ordenación de Bologna, 2008a), caracterizadas por su tamaño mayor, coloración corporal azulada o negra, normalmente con pilosidad muy reducida, punteado y rugosidad variables y antenómeros poco alargados, generalmente subcónicos. En el ámbito del grupo de M. rugosus, Bologna (1988) diferencia a su vez dos subgrupos; el subgrupo A, que agrupa especies con tegumento corporal negro brillante u opaco, pilosidad corporal negra (en algunos casos sólo en parte castaña), punteado de la cabeza y pronoto y rugosidad elitral en general bien marcadas, con 15 especies (una de ellas, Meloe affinis Lucas, 1847, con cuatro subespecies); y el subgrupo B cuyas especies, que se cifran en 9 , presentan el tegumento negro opaco, frecuentemente con las patas y antenas de color pardo-castaño al menos en las regiones apicales, pilosidad clara (pardo-amarillenta), cabeza finamente punteada, y rugosidad elitral reducida en la mayor parte de ellas (Bologna, 1988, 1991, 1994a).

Hasta la caracterización de las especies euromediterráneas del grupo de M. rugosus efectuada por Bologna (1988), reinaba una secular confusión taxonómica entre los representantes del mediterráneo occidental, especialmente los del subgrupo B y más concretamente entre Meloe nanus Lucas, 1847 , Meloe murinus Brandt et Erichson, 1832, Meloe baudueri Grenier, 1863 y Meloe flavicomus Wollaston, 1854, así como con Meloe ganglbaueri Apfelbeck, 1907 (incluido en el subgrupo A, pero con algunos caracteres intermedios entre ambos subgrupos), dando lugar a interpretaciones erróneas de las mismas (como ya apuntó Pardo Alcaide, 1951: 252), por lo que numerosos registros previos han de tomarse con cautela y requieren una revisión crítica, ya avanzada por Bologna (1988, 1991, 1994a, 1994b).

En este trabajo se describe una especie nueva de Meloe del subgénero Eurymeloe, del grupo de $M$. rugosus, que se adscribe al subgrupo B (sensu Bologna, 1988), a partir de ejemplares procedentes del noroeste de Marruecos, concretamente del Rif occidental (Parque Nacional de Talassemtane, dorsal caliza rifeña), en hábitat forestal hiperhúmedo de montaña. Este nuevo taxon es fenéticamente afín a $M$. nanus, $M$. baudueri y $M$. flavicomus, pero diferenciada de ellas tanto por caracteres morfológicos externos como del edeago. De igual forma, se exponen los caracteres discriminantes entre el nuevo taxon y las demás especies mediterráneas del subgrupo en el que se integra.

\section{Material y métodos}

La descripción de la especie nueva de Eurymeloe de Marruecos está basada en el estudio de un total de 15 ejemplares, 8 hembras y 5 machos conservados en seco, más otros dos ejemplares conservados en etanol, integrantes de la serie-tipo, colectados en idéntica localidad y fecha. Las instituciones y colecciones en las que se ha depositado el material tipo de esta nueva especie o de las que se ha estudiado material de comparación, y sus respectivas siglas, son las siguientes: Museo Nacional de Ciencias Naturales, Madrid, España (MNCN); Museu de Zoologia, Barcelona, España (MZB); Natural History Museum, Londres, Reino Unido (NHM); colección M.A. Bologna, en la Universidad de "Roma Tre", Roma, Italia (MAB); y colección J.L. Ruiz, Ceuta, España (JLR). Asimismo, como material de comparación de los taxones específicos del subgrupo en el que se integra la nueva especie, se han estudiado ejemplares de las especies que a continuación se indican y cuyos datos se relacionan en el Apéndice: M. baudueri (12 ejemplares), M. flavicomus (5 ejemplares), M. murinus (7 ejemplares), Meloe nanus (24 ejemplares) y M. pallidicolor (10 ejemplares, sintipos). Parte de este material fue estudiado por M.A. Bologna y porta sus etiquetas de identificación. La serie tipo de $M$. (E.) pallidicolor Escalera 1914 fue examinada debido a que esta especie ha sido adscrita al subgrupo B o subgrupo de M. murinus (véase Bologna, 1988) y, además, su área de distribución se centra en la costa atlántica de Marruecos. El resto de las especies del subgrupo B, M. (E.) kandaharicus Kaszab, 1958, M. (E.) marianii Kaszab, 1983, M. (E.) omanicus Kaszab, 1983, M. (E.) otini Peyerimhoff, 1949, M. (E.) punjabensis Kaszab, 1958, M. (E.) saharensis Chobaut, 1898 y M. (E.) vignai Bologna, 1990, están alejadas geográficamente de la nueva especie y son fenéticamente muy distintas. Los caracteres discriminantes respecto a esta última han sido extraídos de las descripciones originales y referencias bibliográficas citadas en el apartado Discusión.

El estudio morfológico se efectuó sobre ejemplares conservados en seco, mediante observación 
con estereomicroscopio. Las genitalias, extraídas a ejemplares frescos (especie nueva) o secos previamente rehidratados (material de comparación), se montaron en cartulinas con resina DMHF, las cuales se pincharon con su respectivo ejemplar. Las medidas se efectuaron con micrómetro acoplado a uno de los oculares y los dibujos con cámara clara. La longitud total de los ejemplares se ha tomado desde el extremo de la frente (en posición dorsal) hasta el extremo del abdomen. La medida de la longitud del pronoto se ha tomado desde el nivel del extremo de los lóbulos del margen posterior hasta el punto medio del margen anterior; la medida de la anchura pronotal corresponde a la máxima entre los márgenes laterales. Los dos paratipos conservados en etanol no se midieron para evitar un posible deterioro de los tejidos. Las fotografías se efectuaron a ejemplares tanto en vivo (paratipos) como secos (holotipo) con cámara digital. La terminología de las diversas partes de la genitalia masculina se basa en la expresada por Bologna $(1988,1991)$. Puesto que los estadíos larvarios de la especie nueva son desconocidos, la discusión sobre la posición taxonómica y afinidades se efectúa exclusivamente a partir de caracteres morfológicos imaginales. Para la exposición del área de distribución y registros de las especies del subgrupo de $M$. murinus, especialmente de las mediterráneo occidentales, se han tenido en cuenta únicamente los trabajos publicados con posterioridad a la revisión de Bologna (1988), debido a la confusión taxonómica reinante previa a dicha revisión, a no ser que se especifique lo contrario.

\section{Resultados}

\section{Meloe (Eurymeloe) gomari sp. nov.}

MATERIAL TIPO: HOLOTYPUS: 1 macho (conservado en seco), etiquetado: "Yebel Taloussisse, P.N. Talassemtane, prov.

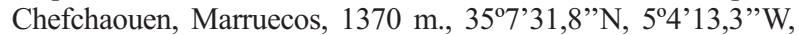
27-I-2008, J.L. Ruiz leg." (etiqueta blanca, impresa); "Holotypus, Meloe (Eurymeloe) gomari, Ruiz \& Ga-París des. 2009” (etiqueta roja, impresa). Depositado en col. MNCN.

PARATYPI: 4 machos, 8 hembras (conservados en seco) y otros dos ejemplares (conservados en etanol) (Fig. 1) etiquetados: "Yebel Taloussisse, P.N. Talassemtane, prov. Chefchaouen, Marruecos, 1370 m., 35 7' 31,8”N, 5' 4' 13,3”W, 27-I-2008, J.L. Ruiz leg." (etiqueta blanca, impresa); "Paratypus, Meloe (Eurymeloe) gomari, Ruiz \& Ga-París des. 2009" (etiqueta roja, impresa). Depositados en: ocho paratipos (dos de ellos en etanol) en col. MNCN; un paratipo en col. NHM; un paratipo en col. MAB; cuatro paratipos en col. JLR.

DESCRIPCIÓN DEL HOLOTIPO. Longitud total: 11,7 mm. Anchura máxima, situada en el ápice de los élitros: $6.05 \mathrm{~mm}$. Aspecto relativamente estilizado, con antenas y patas gráciles (Figs. 2A-C). Coloración general del cuerpo negra, mate, con cierta tonalidad grisácea, únicamente con los artejos X y XI de las antenas tornándose castaño oscuro. Pilosidad corporal blanquecino-amarillenta, cortísima y muy fina, especialmente la de la cabe$\mathrm{za}$, antenas, pronoto, élitros y terguitos abdominales, más larga la del clípeo, labro, patas y esternitos abdominales; sin formar mechones o agrupaciones de pelos claramente perceptibles.

Cabeza (Figs. 2A, 2D) más ancha que el pronoto, con tegumento negro opaco y microrreticulado entre los puntos, con las sienes ampliamente redondeadas y apenas dilatadas, débilmente truncada por detrás; máxima anchura un poco por detrás del medio, presentando una relación "anchura máxima/longitud desde la sutura clípeo-frontal al vértex" (en visión frontal) de 1.35, con un fino y poco impreso surco longitudinal medio que se prolonga desde el disco de la frente (a nivel del borde posterior de los ojos) hasta el vértex. Frente ligeramente convexa, con una leve depresión central a nivel del inicio del surco mediano, apenas deprimida por encima de las inserciones antenales que son marcadamente elevadas; anchura mínima entre los ojos de $1.6 \mathrm{~mm}$. Ojos pequeños pero protuberantes, escasamente escotados a nivel de las inserciones antenales. Sutura clípeo-frontal neta aunque poco profunda. Clípeo negro mate, transverso, 1.74 veces más ancho que largo, subtrapezoidal, con los lados redondeados, plano en la mayor parte de su superficie, con el tercio anterior membranoso de coloración grisáceo amarillenta. Labro superior negro, 1.9 veces más ancho que largo, profundamente hendido en el centro, formando dos lóbulos redondeados a ambos lados de la escotadura central. Punteado de la cápsula cefálica constituido por puntos relativamente pequeños y bien impresos, subcontiguos en el vértex, donde alcanzan la mayor densidad, y menos densos en la región discal de la frente, sienes y por encima de los ojos. Punteado del clípeo formado por puntos algo más gruesos e impresos que los de la frente, densos, distribuidos por toda su superficie a excepción de la banda membranosa; el del labro similar al del clípeo y restringido a los dos tercios anteriores, concentrado sobre todo en los lóbulos, con el tercio basal apenas punteado. Pilosidad de la cápsula cefálica cortísima y fina, amarillenta, apenas perceptible, semitendida, siguiendo el patrón de distribución del punteado en el que se inserta; la del clípeo y labro más larga y gruesa, semierecta, igualmente siguiendo el patrón de distribución del punteado. Mandíbulas 



Fig. 1.- Facies de ejemplares vivos Meloe (Eurymeloe) gomari sp. nov. (Paratipos: Yebel Taloussisse, P.N. Talassemtane, Marruecos). Nótese la coloración negra mate con tonos grisáceos; la pilosidad amarillo-blanquecina muy corta, que recubre la mayor parte del cuerpo de los ejemplares sin formar mechones, el pronoto transverso, subrectangular, con surco medio bien definido y dos depresiones laterales, y la rugosidad poco pronunciada de los élitros.

Fig. 1.- View of live specimens of Meloe (Eurymeloe) gomari sp. nov. (Paratipi: Yebel Taloussisse, P.N. Talassemtane, Marruecos). Note the dull black coloration with grey hue; the very short, yellow-withish pilosity, which covers most of the specimen and does not form tufts; the subrectangular transverse pronotum with a marked median grove and two longitudinal lateral depressions; and the elytra sligtly rugose. 

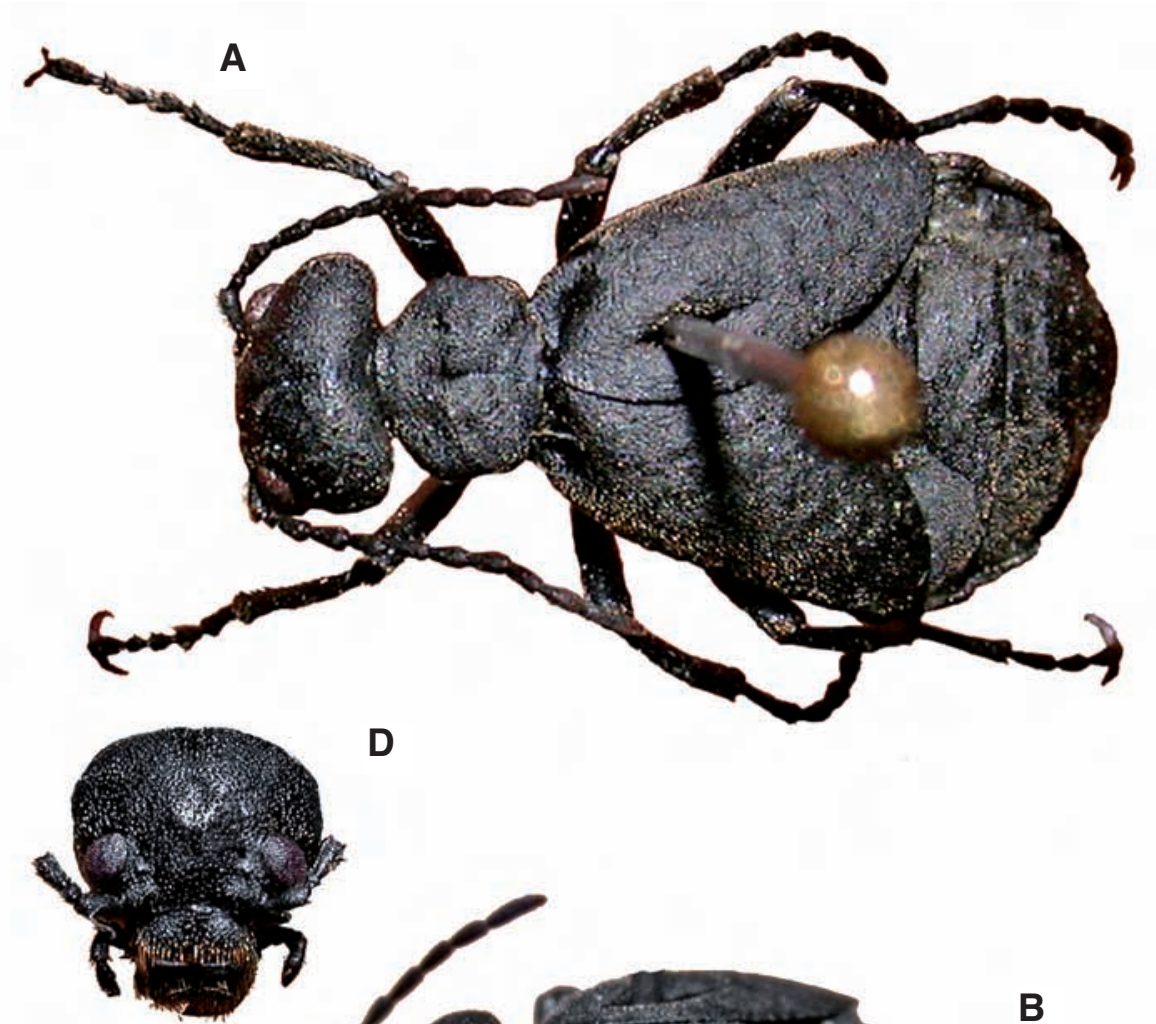

D

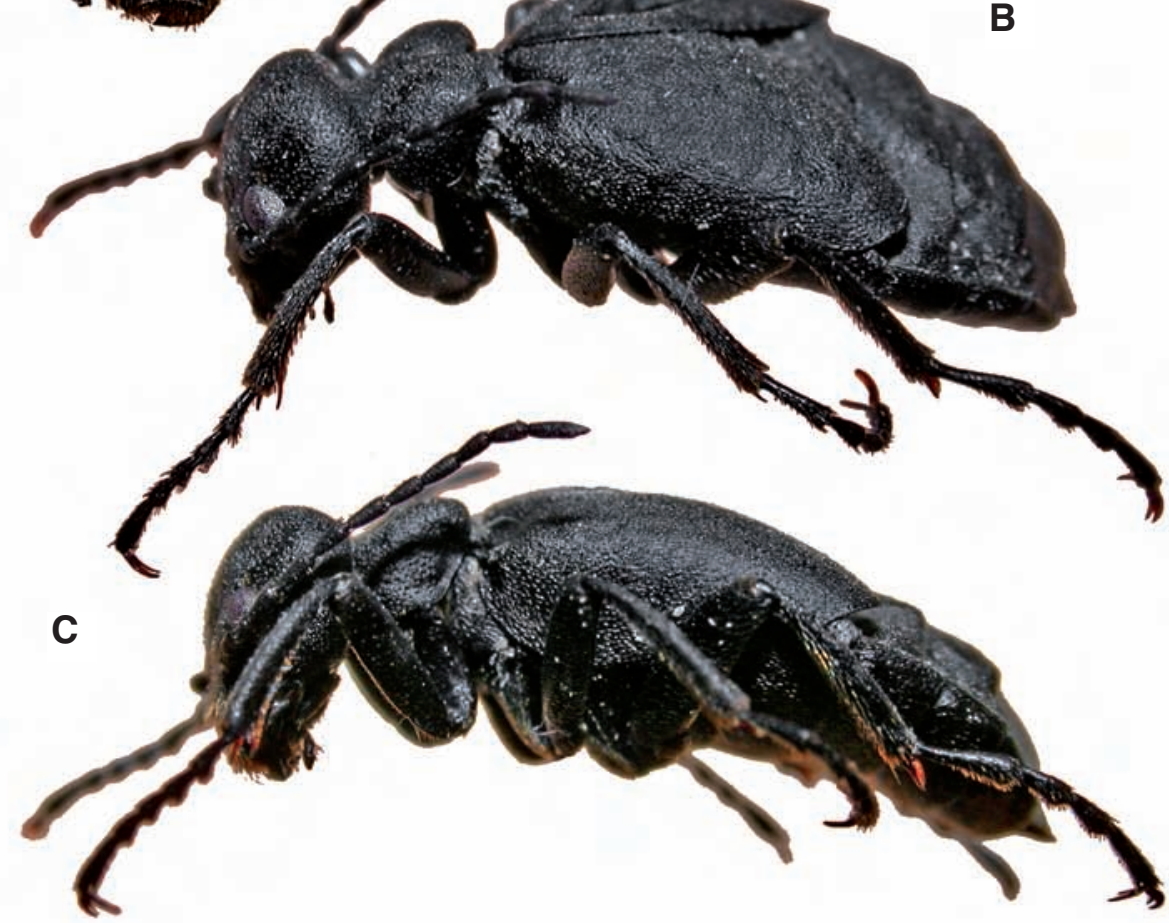

Fig. 2.- Meloe (Eurymeloe) gomari sp. nov. (Holotipo: Yebel Taloussisse, P.N. Talassemtane, Marruecos): Habitus, en visión dorsal (A), dorso-lateral (B) y lateral (C). (Longitud del ejemplar: $11.7 \mathrm{~mm}$ ). Cabeza, en visión frontal (D) (Anchura de la cabeza: $3.1 \mathrm{~mm}$ ).

Fig. 2.- Meloe (Eurymeloe) gomari sp. nov. (Holotypus: Yebel Taloussisse, P.N. Talassemtane, Morocco): a) Habitus, dorsal view (A), dorso-lateral view (B) and lateral view (C). (Specimen length: $11.7 \mathrm{~mm}$ ). Head, frontal view (D) (Head width: $3.1 \mathrm{~mm})$. 

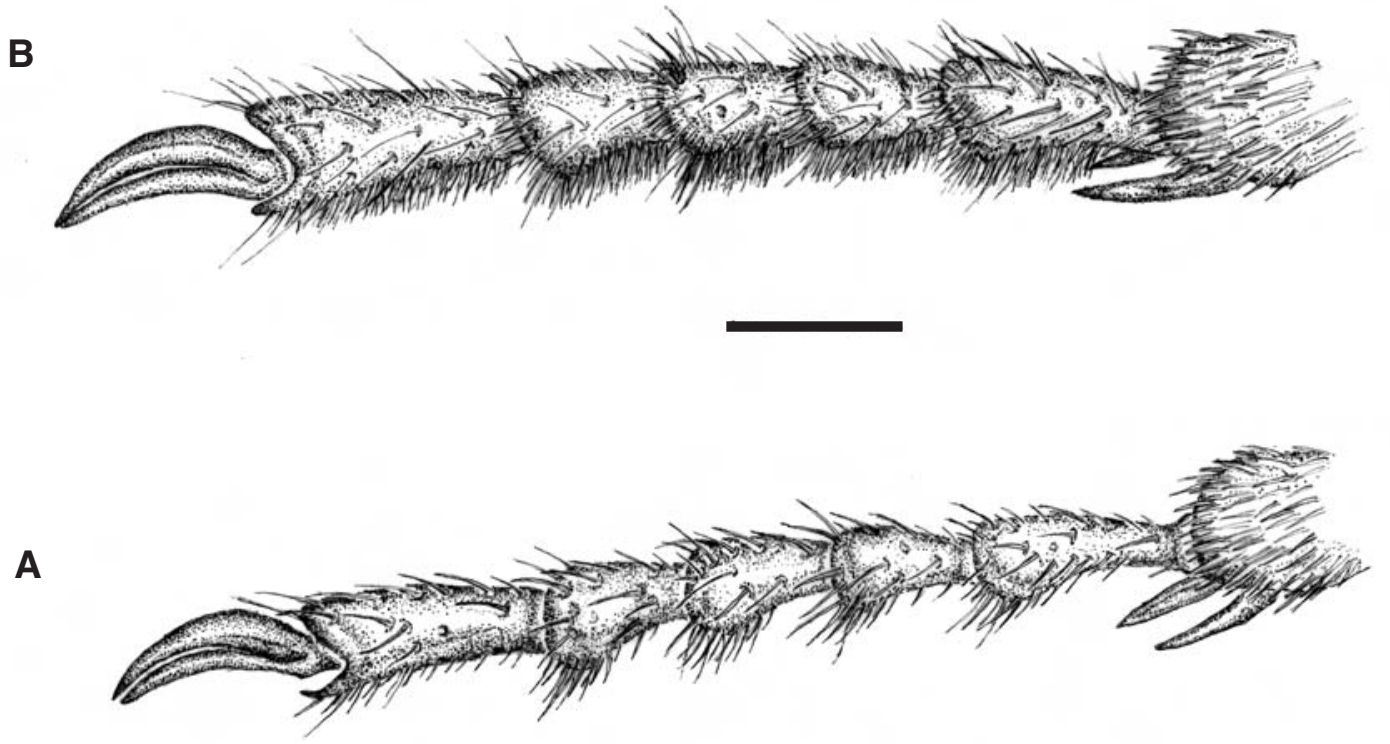

Fig. 3.- Tarso anterior izquierdo de: A) Meloe (Eurymeloe) gomari sp. nov. (Holotipo: Yebel Taloussisse, P.N. Talassemtane, Marruecos); B) Meloe (Eurymeloe) baudueri (Beni-Msuar, Marruecos). Escala: 0.5 mm (J.L. Ruiz del.).

Fig. 3.- Left fore tarsus of: A) Meloe (Eurymeloe) gomari sp. nov. (Holotypus: Yebel Taloussisse, P.N. Talassemtane, Morocco); B) Meloe (Eurymeloe) baudueri (Beni-Msuar, Morocco). Scale bar: $0.5 \mathrm{~mm}$ (J.L. Ruiz del.).

negras, mates, de conformación normal, con la mitad posterior de su margen externo densamente punteado-piloso, con puntos y pelos similares a los del labro. Palpos maxilares alargados, lustrosos, con el artejo distal castaño oscuro, subcilíndrico y truncado en el ápice, que muestran una pilosidad escasa y más corta que la del labro. Palpos labiales de color castaño oscuro, cortos, con el artejo distal securiforme, muy dilatado.

Antenas de 11 artejos, negras, mates, a excepción de los tres primeros antenómeros que son ligeramente lustrosos y los artejos IX a XI que se tornan paulatinamente un poco más claros (castaño oscuro), de aspecto grácil y largas, extendidas hacia atrás alcanzan el primer tercio elitral; artejos alargados, a excepción del II. Antenómero I subcónico, estrechado por delante de la inserción basal, 2.3 veces más largo que ancho y 2.5 veces más largo que el siguiente; II muy corto, marcadamente ensanchado en la mitad apical, aproximadamente igual de ancho que largo; III subcilíndrico, 3 veces más largo que ancho, ligeramente ensanchado en la región distal, de similar longitud al siguiente; IV subcilíndrico, 2.4 veces más largo que ancho, débilmente ensanchado en el ápice; $\mathrm{V}$ un poco más corto que el cuarto, subcilíndrico, 2.1 veces más largo que ancho, escasamente ensanchado en el ápice; VI y VII de longitud similar, subcilíndricos, ligeramente más largos que el $\mathrm{V}$ y unas 2.5 veces más largos que anchos; VIII, IX y $\mathrm{X}$ subiguales, cilíndricos, de lados subparalelos, unas 2.5 veces más largos que anchos; XI alargado, 1.5 veces más largo que el anterior, de lados subparalelos excepto en la zona apical donde se estrecha débilmente para terminar en punta redondeada. Antenómeros I a III con pilosidad amarillenta, semitumbada y recta, doble de larga que la de la cabeza y similar a la de las caras laterales de los tarsómeros, relativamente densa; IV con algunos escasos pelos similares a los de los artejos anteriores; IV a XI con pilosidad cortísima, poco perceptible.

Pronoto (Figs. 2A, 2B) negro mate, con cierta tonalidad grisácea $\mathrm{y}$ el tegumento fuertemente micorreticulado entre los puntos, transverso, subrectangular, presentando una relación "anchura/longitud" de 1.63 y máxima anchura a nivel de los ángulos laterales, que son redondeados; región dorsal en suave declive hacia delante en el cuarto anterior y en declive abrupto hacia atrás en el cuarto posterior, con un fino pero profundo surco longitudinal mediano que recorre casi toda su longitud, con un área elevada y convexa que se extiende por cada uno de los dos cuartos centrales de cada lado, de límites difusos, en cuyas respectivas regiones 
centrales se aprecian dos tenues depresiones longitudinales totalmente punteadas, más marcadas hacia delante; margen posterior fuertemente escotado en medio; margen anterior en arco poco marcado; márgenes laterales subparalelos, apenas convergentes hacia atrás en los tres cuartos posteriores, fuertemente convergentes hacia delante en su cuarto anterior. Punteado del pronoto constituido por puntos similares a los del vértex, pero algo menos impresos, muy densos, subcontiguos, que le imprimen un aspecto subrugoso, casi uniformemente repartidos, con la menor densidad justo por detrás del margen anterior y en las leves depresiones laterales. Pilosidad pronotal cortísima, fina y escasamente perceptible, blanquecino-amarillenta, semitendida y curvada, siguiendo el patrón del punteado en el que se inserta; la de la región anterior dirigida hacia atrás, la de los lados y disco dirigida hacia el centro y la de la región posterior dirigida hacia delante. Propleuras mates, casi impunteadas, con escasos puntos dispersos poco impresos y pilosidad igualmente escasa. Mesonoto con el borde posterior subrectilíneo, con una escotadura estrecha en el medio y un fino surco longitudinal mediano, fuertemente punteado en toda su superficie excepto en el surco, con pilosidad amarillenta, corta y dirigida hacia atrás. Metanoto oculto por la base de los élitros. Prosterno poco prolongado hacia atrás, terminado en punta redondeada, con punteado similar al del vértex. Mesosterno normalmente conformado, escasamente prolongado entre las coxas medias, terminado en punta roma, y con la superficie con arruguitas longitudinales. Metasterno fuertemente hendido en forma de $\mathrm{V}$ en el centro de su margen posterior.

Elitros montados en la base (el izquierdo sobre el derecho), dehiscentes, relativamente alargados, sobrepasando un poco la mitad anterior del abdomen, de color negro mate, con una ligera tonalidad grisácea, con el tegumento fuertemente microrreticulado; sin rugosidad evidente, sólo con unas muy tenues gibosidades onduladas de límites difusos y más o menos longitudinales; punteado apenas evidente, confundido con el microrreticulado tegumentario; pilosidad cortísima, muy fina, amarillenta, poco perceptible, con pelos semicurvados y tumbados hacia atrás, poco densa y uniformemente repartida por la superficie elitral.

Región dorsal del abdomen de color negro mate. Terguitos abdominales con punteado fino y difuso, prácticamente confundido con el microrreticulado del tegumento; placas esclerotizadas centrales casi indistintas, algo más evidentes en los tres últimos terguitos, donde son ligeramente lustrosas; pilosi- dad blanquecino-amarillenta, similar a la de los élitros, cortísima y muy fina, poco densa, sin formar mechones o agrupaciones de pelos ni siquiera en el borde posterior de los terguitos, uniformemente repartida a excepción de una estrecha banda centrolongitudinal depilada en las áreas esclerotizadas; el último terguito presenta en su margen posterior pelos dorados entre tres y cuatro veces más largos que los del resto de terguitos, rectos, más densos y agrupados en el extremo apical. Esternitos abdominales negros, sedosos, más bien brillantes, con punteado difuso, poco impreso y denso, formando pequeñas arrugas transversales; pilosidad constituida por pelos amarillentos, relativamente densos, similares a los del último terguito, tumbados y prácticamente aplicados contra la superficie; último esternito fuertemente escotado en el ápice y con una depresión central bien marcada y sin pelos.

Patas de aspecto grácil, negras, con el extremo distal de los tarsómeros tornándose castaño oscuro, éstos brillantes; pilosidad amarillenta, algo más oscura en el extremo de las tibias y un poco más larga y gruesa que la de los esternitos abdominales, tumbada, siendo más densa en las tibias que en los fémures. Tarsos alargados, ligeramente más largos que las tibias; tarsómeros I a IV (Fig. 3A) que portan en su cara inferior un cepillito poco conspicuo de pelos castaño-amarillentos cortos y erectos, distribuidos únicamente en la mitad distal, y en la proximal sólo algunos pelos dispersos, el $\mathrm{V}$ no presenta un cepillo bien definido, únicamente algunos pelos alineados pero no apretados; pilosidad de las caras superior y laterales de los tarsómeros similar a la de las tibias, pero con pelos más cortos. Espinas de las tibias anteriores de longitud algo mayor que un tercio de la del primer tarsómero, subiguales, acuminadas hacia el ápice, ligeramente curvadas y terminadas en punta roma; espinas mesotibiales similares a las de las protibias, subiguales; espinas de las metatibias con la interna ligeramente más corta, un poco curvada y algo más gruesa en la base que las de las mesotibias; la externa más gruesa, en forma de cuchara poco ensanchada, cortada en bisel en su cara superior. Uñas normales, débil y regularmente curvadas, con ambos lóbulos de longitud similar.

Edeago (Figs. 4A-C) de aspecto estilizado y relativamente corto; falobase con longitud similar a la de los parámeros, más ancha que estos en visión dorsal, apenas convexa dorsalmente; parámeros más bien estrechos en visión lateral, netamente acuminados y estrechos en su tercio distal en visión dorsal, con los lóbulos paramerales terminados en punta y curvados en visión lateral, presentado una 
A

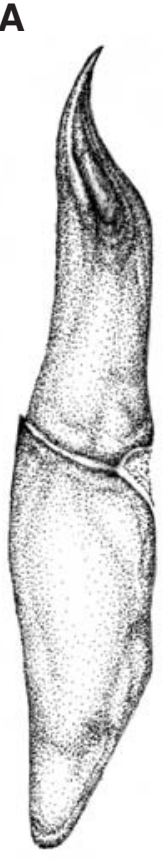

D



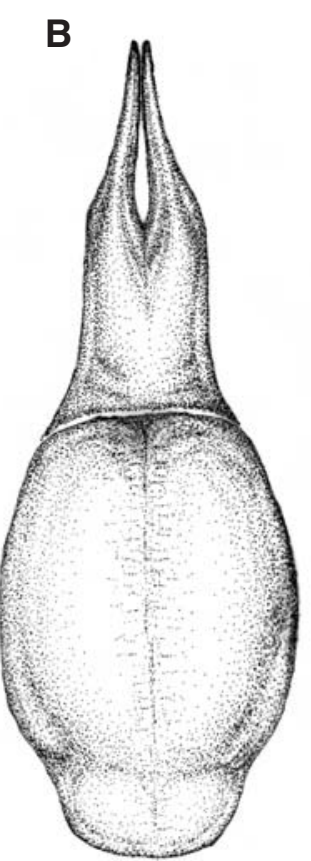

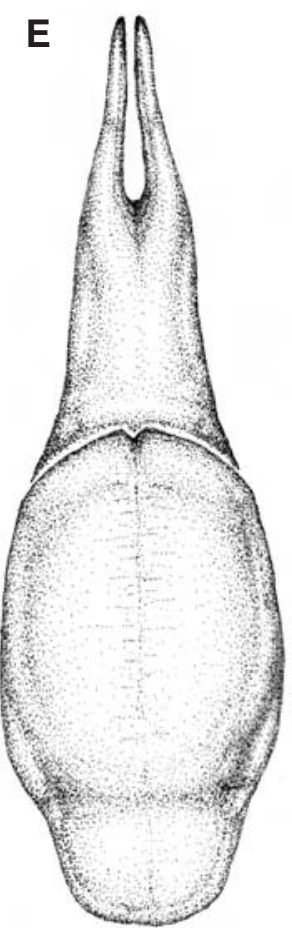

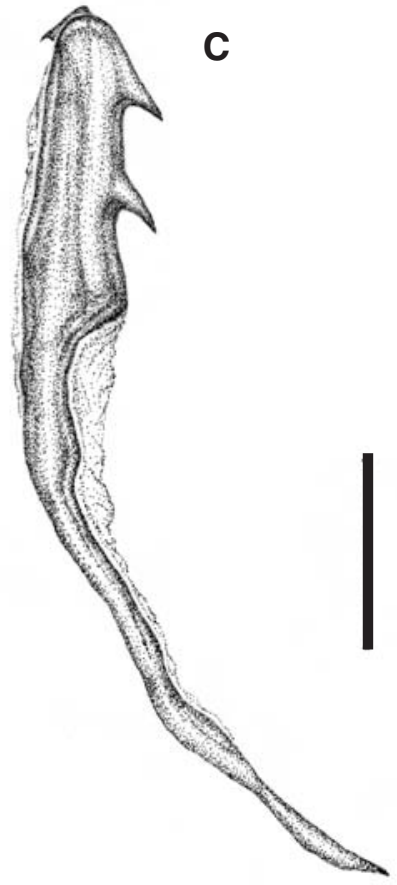

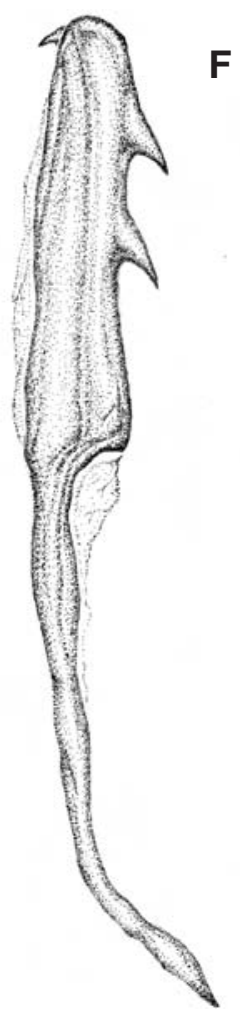

Fig. 4.- Genitalia masculina de: Meloe (Eurymeloe) gomari sp. nov. (Holotipo: Yebel Taloussisse, P.N. Talassemtane, Marruecos): tegmen, en visión lateral (A) y dorsal (B); lóbulo medio, en visión lateral (C). Meloe (Eurymeloe) baudueri (Beni-Msuar, Marruecos): tegmen, en visión lateral (D) y dorsal (E); lóbulo medio, en visión lateral (F). Escala: 0.5 mm (J.L. Ruiz del.).

Fig. 4.- Male genitalia of: Meloe (Eurymeloe) gomari sp. nov. (Holotypus: Yebel Taloussisse, P.N. Talassemtane, Morocco): tegmen, lateral view (A) and dorsal view (B); C) median lobe, lateral view. Meloe (Eurymeloe) baudueri (Beni-Msuar, Morocco): tegmen, lateral view (D) and dorsal view (E); median lobe, lateral view (F). Scale bar: $0.5 \mathrm{~mm}$ (J.L. Ruiz del.). 
depresión bien patente en sus márgenes laterales; lóbulo medio alargado y curvado en su mitad proximal, con dos fuertes dientes ventrales, subiguales, apenas curvados en su extremo, netamente separados entre sí, el distal un poco más cerca del ápice que del diente proximal; gancho dorso-apical (uncus) pequeño e inclinado, apenas visible.

VARIABILIDAD. La hembra es similar al macho, excepto el último esternito abdominal que es redondeado en el ápice, sin escotadura apical y sin depresión central, uniformemente piloso. Valvífera de la hembra como en Fig. 6. La variabilidad observada en los ejemplares de la serie tipo es escasa y afecta de manera perceptible a los siguientes caracteres: longitud total, entre 10.7$16.1 \mathrm{~mm}($ media $=12.6 \mathrm{~mm} ; \mathrm{n}=13)$; relación "anchura/longitud" del pronoto, entre 1.58-1.7 (media $=1.63 ; \mathrm{n}=13$ ); longitud de las antenas con pequeñas variaciones, en general un poco más largas en los machos; color de los artejos antenales, que en algunos ejemplares se torna castaño a partir del VI o VII, con el XI castaño-rojizo; depresión longitudinal de los lados del pronoto más o menos marcada y con extensión algo variable, pero en general bastante homogénea; rugosidad elitral, un poco más marcada en algunos ejemplares (los de tamaño mayor), aunque siempre débil (Fig. 1); pilosidad de los esternitos abdominales, ligeramente más densa en algunos ejemplares, aunque en todo caso cortísima y curvada; cepillos de la cara inferior de los tarsómeros, que se reduce en algunos casos al cuarto apical y se hace menos densa, $\mathrm{y}$ en un ejemplar de pequeño tamaño $(10.7 \mathrm{~mm})$ casi desaparecen, siendo sustituida por pelos no apretados. La genitalia masculina es muy uniforme, con escasa variación en la longitud y curvatura de los lóbulos paramerales.

ETIMOLOGía. El nombre gomari corresponde a la trascripción latina del vocablo bereber (tamazight) referente al habitante de la Gomara (o Ghomara), región o comarca del Rif centro-occidental, en la que se ubica la localidad tipo de la especie, en parte bereberófona en la actualidad (Behnstedt, 2002). Los límites de la región de Gomara son imprecisos y variables según autores (e.g. El Gharbaoui, 1981; Hajjarabi, 1991; Nogué \& Villanova, 1999) y, a grandes rasgos, se extiende desde el río Lau (Oued Laou) al oeste hasta el río Ouringa (Oued Ouringa) al este, con límite norte en la costa mediterránea y sur en las estribaciones meridionales de la cordillera rifeña, abarcando las montañas calizas de la región de Xauen (Chefchaouen).
DisTRIBUCIÓN Y HÁBITAT. Hasta el momento, $M$. gomari sólo se conoce de la localidad tipo, situada en pequeños llanos entre montañas, a $1370 \mathrm{~m}$ de altitud, en la falda noreste del Yebel Taloussisse (altitud máxima: $2005 \mathrm{~m}$ ), a escasa distancia (3.5 $\mathrm{km}$ al este) del Yebel Talassemtane, en el Parque Nacional de Talassemtane (Rif occidental, provincia de Chefchaouen), que a su vez ha sido integrado en la Reserva de la Biosfera Intercontinental del Mediterráneo Andalucía (España)-Marruecos (véase Benabid, 2000; Molina Vázquez, 2006).

Geológicamente, esta localidad se ubica en la zona externa de la Dorsal Caliza Rifeña, en el dominio del manto del Yebel Tissouka y más concretamente en la unidad del Taloussisse, con materiales de tipo dolomías tabulares más o menos disgregadas o alteradas (pulvilunares) (Wildi, 1979; Sanz de Galdeano, 1997). Desde un punto de vista bioclimático se sitúa en el horizonte supramediterráneo, con un ombrotipo hiperhúmedo (según clasificación de Rivas-Martínez, 1987; véase Benabid, 1984, 1985, 2000), que sobrepasa los $1600 \mathrm{~mm}$ de precipitación media anual [en la cercana estación de Bab Taza, a $880 \mathrm{~m}$ de altitud se ha registrado una precipitación media anual de $1482 \mathrm{~mm}$, aunque en zonas de mayor altitud y más septentrionales, como la que nos ocupa, aumenta la pluviosidad (Benabid, 1982)]. La vegetación de la zona, a grandes rasgos, está constituida por formaciones arbóreas mixtas más o menos abiertas de Pinus pinaster hamiltonii (Ten.) Huguet del Villar y de Pinus nigra mauritanica (Maire et Peyerimh.) Heywood, y a cota inmediatamente superior (a escasos 50-80 m por encima) aparecen formaciones de pinsapo o abeto marroquí, Abies maroccana Trabut [o Abies pinsapo maroccana (Trabut) Emberger et Maire, según autores (véase Charco, 1999)], formaciones forestales realmente singulares y exclusivas de estos relieves dolomíticos rifeños.

Fitosociológicamente, la vegetación señalada sería transicional e introgresiva entre las asociaciones denominadas Pino maghrebianae-Ulicetum funkii (integrada en la alianza Lithodoro-Ulicion funkii) y Pino maghrebianae-Genistetum gomaricae (alianza Pseudoscabioso grossii-Origanion grossii), ambas estrictamente dolomitícolas, donde constituyen asociaciones edafófilas, pedoclimácicas, la primera de menor altitud (aproximadamente entre 700-1400 m) y la segunda sustituye a aquella a altitud superior (1400-1700 m), situándose a partir de los $1400 \mathrm{~m}$ de altitud en la serie húmedahiperhúmeda del abeto marroquí (Paeonio maroccanae-Abietetum maroccanae) (véase Benabid, 1982, 1984, 2000; Quézel et al., 1988; Benabid \& Fennane, 1994; Charco, 1999). Como 
A

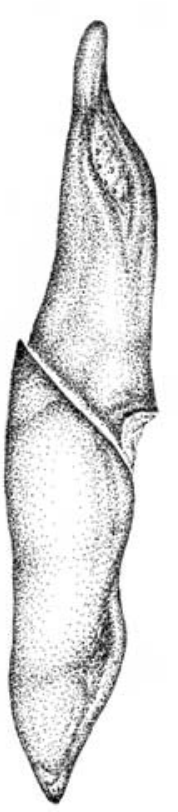

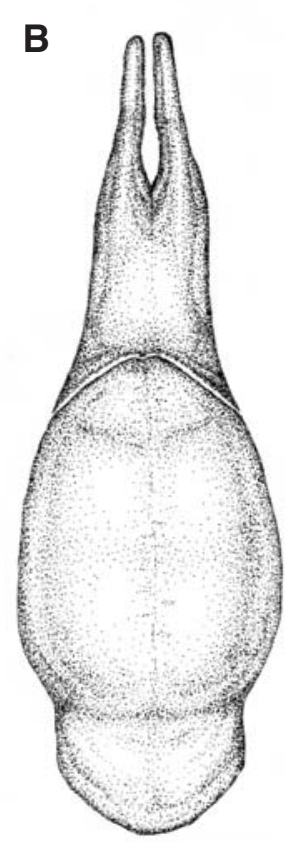

C

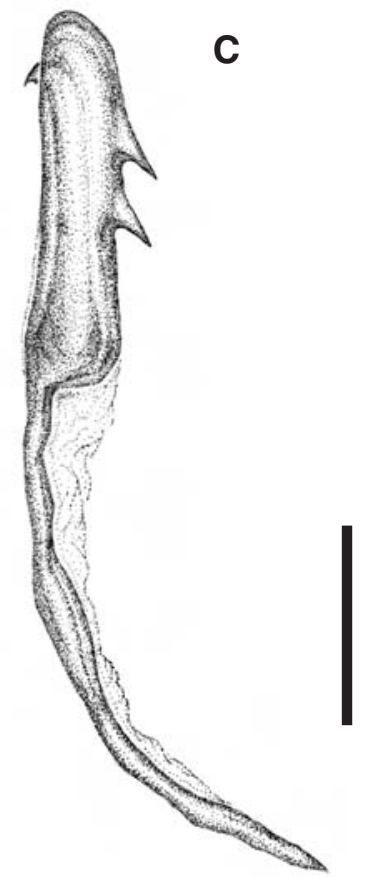

Fig. 5.-Meloe (Eurymeloe) nanus (Daya, Alicante, España): tegmen, en visión lateral (A) y dorsal (B); lóbulo medio, en visión lateral (C). Escala: $0.5 \mathrm{~mm}$ (J.L. Ruiz del.).

Fig. 5.- Meloe (Eurymeloe) nanus (Daya, Alicante, Spain): tegmen, lateral view (A) and dorsal view (B); median lobe, lateral view (C). Scale bar: $0.5 \mathrm{~mm}$ (J.L. Ruiz del.).

elementos florísticos característicos de estas asociaciones cabría citar, además de las especies de coníferas arriba reseñadas, a Ulex parviflorus Pourr., Genista cephalanta demnatensis (Murb.) C. Raynaud, Lithodora maroccana I. M. Johnst., Origanum grossii Pau et Font Quer, Thymelea tartonraira (L.) All., Phlomis caballeroi Pau, Ptilostemon riphaeum Maire, Quercus rotundifolia Lamk., Cistus albidus L. y Juniperus oxycedrus L.

Los ejemplares de Meloe gomari fueron hallados inactivos bajo piedras y bajo cortezas de pino dispersas por el suelo, en las horas centrales de la jornada, en pleno invierno (finales de enero), coincidiendo con el espectro fenológico habitual en la mayor parte de las especies de Eurymeloe (e.g. Bologna, 1988, 1991). Bajo una corteza de pino de mediano tamaño $(50 \times 27 \mathrm{~cm})$ se encontró una agrupación de seis individuos de ambos sexos, muy juntos, que quizás podría ser indicio de un comportamiento de tipo "hilltop aggregation" o agregación reproductiva, estrategia que facilita el encuentro entre sexos en especies en las que uno o ambos sexos se encuentran ampliamente distribuidas espacial o temporalmente, al incrementar la posibilidad de contacto entre los mismos (Shields, 1967; Catts, 1979; Alcock, 1983). Este tipo de agregaciones parece ser excepcional en meloidos no voladores y únicamente ha sido observada en la especie americana Meloe (Meloe) strigulosus Mannerheim, 1852 (García-París \& Parra-Olea, 2003).

\section{Discusión}

Meloe gomari se caracteriza en el seno de Eurymeloe por los siguientes rasgos diagnósticos: tamaño pequeño-mediano; coloración corporal negro mate con cierta tonalidad grisácea; pilosidad blanquecino-amarillenta, cortísima, curvada y poco densa en cabeza, pronoto, élitros y terguitos abdominales, donde no forma agrupaciones ni mechones; pronoto con surco medio longitudinal, transverso, subrectangular, con relieves bien marcados y sendas depresiones longitudinales a ambos 
lados del centro; punteado de cabeza y pronoto formado por puntos bien impresos y subconfluentes; élitros apenas rugosos; tarsómeros con agrupaciones de pelos (cepillos) de la cara inferior poco desarrolladas, distribuidos sólo entre la mitad y el tercio distal; edeago con la vaina parameral corta, lóbulos paramerales curvados con depresiones laterales y terminados en punta aguda, y lóbulo medio robusto con el diente distal de la zona ventral cercano al ápice.

De acuerdo con los grupos de especies establecidos por Bologna (1988) en el ámbito de Eurymeloe, $M$. gomari se integra claramente en el grupo I o grupo de $M$. rugosus, pues presenta la coloración corporal negra, un tamaño mediopequeño $(10.7-16.1 \mathrm{~mm}$; media $=12.6 \mathrm{~mm}, \mathrm{n}=13)$, pilosidad clara, punteado bien marcado (al menos en cabeza y pronoto) y antenas con los artejos alargados y subcilíndricos. A su vez, en el seno de esta agrupación, se adscribe al subgrupo B o subgrupo de M. murinus (sensu Bologna, 1988, 1990, 1991, 1994a, 1994b) por mostrar el tegumento negro opaco, mate, la pilosidad corporal amarillenta, la cabeza con punteado relativamente fino, la rugosidad elitral casi ausente y el extremo de las antenas castaño oscuro.

El subgrupo de $M$. murinus reúne hasta el momento a un total de 12 especies, de las cuales siete se encuentran en la cuenca mediterránea (Bologna, 1988): M. (E.) murinus, de amplia distribución mediterránea, que incluye todo el norte de África, sur de la Península Ibérica, Sicilia, Cerdeña y Creta (Bologna, 1988, 1991, 1994a, 1994b, 2008a); M. (E.) pallidicolor Escalera, 1909, propia de la región suroccidental atlántica de Marruecos, entre Essaouira (= Mogador, loc. typ.) y Casablanca (Escalera, 1909, 1914; Cros, 1939; Kocher, 1956, 1964; Bologna, 1988, 2008a); M. (E.) flavicomus, endemismo macaronésico, conocido del archipiélago de Madeira (Porto Santo, Madeira, Desertas), Islas Selvagens e islas occidentales y centrales del archipiélago canario (El Hierro, La Palma, La Gomera, Tenerife y Gran Canaria) (Bologna, 1988, 1994a, 2008a; Bologna \& Marangoni, 1990; Machado \& Oromí, 2000; García, 2008); M. (E.) nanus, distribuida por la mitad sur de la Península Ibérica, la totalidad del norte de África y Oriente próximo (Siria e Israel) (Bologna, 1988, 1991, 2008a; Pérez-Moreno et al., 2003; Ruiz \& GarcíaParís, 2008); M. (E.) baudueri, presente en la Península Ibérica y suroeste de Francia con registros muy escasos y dispersos (Bologna, 1988, 1991; Ruiz et al., 1993; Pérez-Moreno et al., 2003), y recientemente señalada por Bologna (1994a, 2008a) de Marruecos y Argelia (fue citada por Escherich, 1890, de Argel), aunque sin precisar localidades concretas; M. (E.) kandaharicus Kaszab, 1958, de Irán y Afganistán (Kaszab, 1958, 1983; Bologna, 1988, 2008a); M. (E.) marianii Kaszab, 1983, descrita sobre ejemplares de Egipto (localidad-tipo: "Alexandrie") y de Arabia Saudí (Khashm Khafs) y señalada igualmente de Israel (Kaszab, 1983; Bologna, 1988, 2008a; Bologna \& Turco, 2007); $M$. (E.) saharensis Chobaut, 1898, del Sáhara septentrional argelino (loc. typ.: Ghardaia) (Chobaut, 1898; Borchmann, 1917; Bologna, 1990, 1991, 2008a); M. (E.) otini Peyerimhoff, 1949, del Marruecos nororiental (loc. typ.: plaine de Guercif) (Peyerimhoff, 1949; Kocher, 1956; Bologna, 1991, 2008a); M. (E.) vignai Bologna, 1990, de Somalia (Bologna, 1990); M. (E.) omanicus Kaszab, 1983, endémica del este y sureste de la Península Arábiga (Kuwait, Omán y Emiratos Árabes Unidos) (Kaszab, 1983; Bologna, 1988, 2008a; Al-Houty, 2004); y, por último, M. (E.) punjabensis Kaszab, 1958, descrita del Punjab (loc. typ.: Murree Hills, Thobba), en el actual Paquistán (Kaszab, 1958; Bologna, 1988).

En el ámbito de este subgrupo, M. murinus se segrega sin dificultad del resto de las especies, incluida M. gomari, por la conjunción de los siguientes caracteres: cápsula cefálica con las sienes fuertemente dilatadas, lo que le confiere a la cabeza un aspecto ancho y subgiboso; patas y antenas poco alargadas y de aspecto más robusto, estas últimas no alcanzan el tercio anterior de los élitros extendidas hacia atrás, con los antenómeros III a X comparativamente más cortos y ensanchados, a lo sumo dos veces más largos que anchos; pronoto transverso, con los márgenes laterales angulosos hacia delante y macroescultura muy pronunciada; rugosidad elitral ondulada y manifiesta; y edeago con la vaina parameral ancha y corta en visión lateral, y con los lóbulos paramerales a su vez cortos y relativamente anchos en el extremo apical (Bologna, 1988: 244, 273-275, figs. 18, 33, 46; 1991: 330-331, figs. 114g, h, i, j, k, 1). En Marruecos, M. murinus ha sido registrada con certeza únicamente de las localidades de Marraquech y Volubilis (Bologna, 1988), aunque posiblemente la cita de Kocher (1956) referente a Msoum, al oeste de Taza, sea verosímil, puesto que este autor ya advirtió que esta especie había sido confundida por diversos autores y en las colecciones con $M$. (E.) nanus (Kocher, 1956: 70, nota 3); de igual forma, ofrece como dudoso un registro del Anti Atlas: Bled-Akhsas (Kocher, 1938, recogido en Kocher, 1956). 
Meloe pallidicolor se separa de las demás especies del subgrupo por su facies característica y única en el ámbito de Eurymeloe, con la cabeza, antenas, palpos, patas, protórax y élitros de color amarillento pajizo y abdomen pardo rojizo, si bien en algunos individuos la coloración se oscurece en cabeza, protórax y élitros, tornándose rojizo achocolatado, pero conservando el color claro en antenas, patas y palpos (Escalera, 1909; Bologna, 1988). Además del singular patrón de coloración, M. pallidicolor se diferencia de M. gomari por presentar aquella el pronoto un poco más estrecho y alargado, menos transverso, profundamente hendido por una ancha depresión media, la pilosidad corporal más larga y densa, sobre todo en los márgenes posteriores de los terguitos abdominales, y la genitalia masculina con los parámeros comparativamente más alargados y los lóbulos paramerales más cortos y anchos, asimismo, el lóbulo medio presenta los dos dientes ventrales muy próximos entre sí (Escalera, 1909: 242-243; Bologna, 1988: 285-287, figs. 22, 37, 50, 64, 103-106). Meloe pallidicolor sólo se conoce de cuatro localidades en la franja suratlántica de Marruecos: Essaouira (Escalera, 1909, 1914; Kocher, 1956), Azemmour, Casablanca (Kocher, 1964) y Sidi Said Maachou, unos $30 \mathrm{~km}$ al sureste de El Jadida (Bologna, 1988). Se trata de una especie estenotópica exclusiva de dunas costeras y terrenos arenosos subcosteros, donde según Escalera (1909) pasa casi desapercibida debido a su coloración críptica.

La especie macaronésica $M$. flavicomus se separa de $M$. gomari por presentar, entre otros rasgos, el pronoto menos transverso (relación "anchura/longitud" entre 1.39-1.42; media $=1.4, \mathrm{n}=5$ ), subtrapezoidal, con los márgenes laterales sinuados en su mitad posterior, y macroescultura distinta, con dos depresiones oblicuas de límites difusos antero-laterales; punteado pronotal igualmente distinto, con puntos más pequeños y arruguitas transversas entre los puntos en el disco; la pilosidad de la cabeza, pronoto, élitros y abdomen más larga y densa, sobre todo en la región superior abdominal, donde forma agrupaciones en el borde posterior de los terguitos (aunque menos aparentes que en $M$. nanus) y, por último, la genitalia masculina con la vaina parameral más alargada, los lóbulos paramerales más anchos y cortos, sin la curvatura característica de $M$. gomari, y el lóbulo medio con los dientes ventrales más separados del ápice (Bologna, 1988: 276-279, figs. 19, 34, 47, 61, 93-95). Bologna (1994a) indica haber estudiado ejemplares de Eurymeloe de Marruecos, sin especificar localidades, similares a M. flavicomus y M. baudueri, pero que probable-

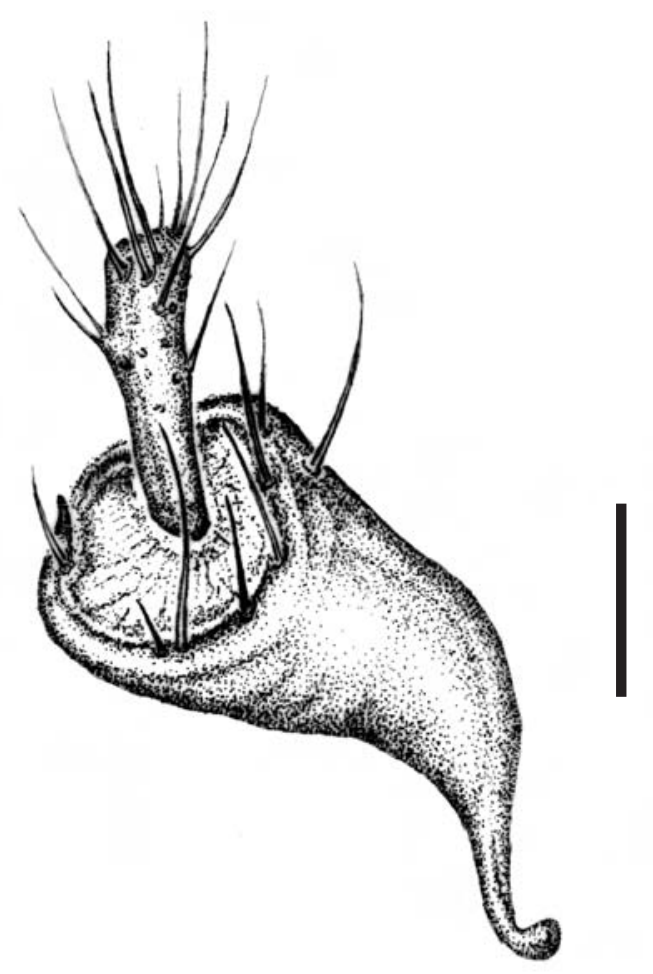

Fig. 6.- Meloe (Eurymeloe) gomari sp. nov. (paratipo hembra: Yebel Taloussisse, P.N. Talassemtane, Marruecos): Valvífera. Escala: $0.3 \mathrm{~mm}$ (J.L. Ruiz del.)

Fig. 6.- Meloe (Eurymeloe) gomari sp. nov. (female paratype: Yebel Taloussisse, P.N. Talassemtane, Morocco): Valvifer. Scale bar: $0.3 \mathrm{~mm}$ (J.L. Ruiz del.).

mente hayan de ser referidos a la segunda; recientemente, este autor no incluye Marruecos en su área de distribución (Bologna, 2008a).

Las especies fenéticamente más próximas a $M$. gomari son $M$. baudueri y $M$. nanus, con las que comparte una facies muy parecida, conformando un grupo morfológicamente homogéneo; no obstante, se separa de ambos taxones por caracteres tanto externos como genitales, que a continuación se indican. Coloración general de $M$. gomari negra con tonalidad grisácea, con patas igualmente negras, mientras que en $M$. nanus y $M$. baudueri es negra con tonalidad castaño-rojiza, sobre todo en las antenas y patas. Pronoto netamente más ancho y transverso en M. gomari, con una relación "anchu$\mathrm{ra} /$ longitud" que varía entre 1.58-1.7 (media = 1.63; $\mathrm{n}=13$ ), mientras que en $M$. nanus oscila entre 1.31.44 (media $=1.38 ; \mathrm{n}=18)$ y en $M$. baudueri entre 1.4-1.56 (media $=1.5 ; \mathrm{n}=9$ ) (según Bologna, 
1988, aproximadamente una vez y media más ancho que largo en esta última especie). Macroescultura pronotal distinta: en M. baudueri, aunque presenta sendas depresiones laterales difusas al igual que en $M$. gomari, la superficie es casi plana, sin fuerte declive posterior ni áreas elevadas laterales convexas y con una escotadura en el margen posterior menos marcada, en tanto que $M$. gomari presenta un fuerte declive posterior y áreas elevadas convexas a ambos lados del surco medio, en cuyas regiones centrales se sitúan sendas depresiones longitudinales difusas; $M$. nanus muestra relieves más marcados en la superficie del pronoto que $M$. baudueri pero menos acusados que en $M$. gomari y no presenta depresiones longitudinales laterales, que en su caso son sustituidas por sendas depresiones antero-laterales de límites difusos, la escotadura basal es más amplia y profunda en $M$. gomari y el surco medio es más largo en ésta, en la que llega casi a alcanzar el margen anterior. Meloe baudueri presenta una pequeña callosidad semicircular de tegumento liso, sin pilosidad ni punteado, que se levanta suavemente en la zona central de las depresiones laterales difusas del pronoto; esta callosidad lisa se presenta ocasionalmente en $M$. nanus, aunque suele estar hundida en una pequeña depresión aislada en lugar de levantada y falta en la totalidad de los ejemplares de $M$. gomari estudiados. Punteado del pronoto más denso y confluente en $M$. gomari, sobre todo en la mitad anterior. Pilosidad de cabeza, pronoto, élitros y terguitos abdominales de M. gomari constituida por pelos extremadamente cortos y curvados, poco perceptibles, más claros (amarillo-blanquecinos) y mucho menos densos, sin formar mechones o agrupaciones patentes en el abdomen; mientras que en $M$. baudueri y M. nanus los pelos son casi el doble de largos, poco curvados o semirectos y mucho más densos, sobre todo en el pronoto, élitros y terguitos abdominales, en estos últimos conforman mechones bien patentes (M. nanus) o agrupaciones más o menos densas en el borde posterior (M. baudueri). Punteado y pilosidad de la cara externa de fémures y tibias más denso en $M$. baudueri y $M$. nanus. Tarsómeros de M. baudueri y M. nanus presentando en su cara inferior un conspicuo cepillo conformado por pelos muy finos, cortos y densos, que se extiende por la práctica totalidad de la superficie inferior de los artejos tarsales, incluido el V; en $M$. gomari dicho cepillo es mucho menos patente, está constituido por pelos castaño-amarillentos más gruesos y menos densos, sólo se extiende entre un tercio y la mitad distal de la cara inferior de los tarsómeros, y falta en el artejo $\mathrm{V}$, donde aparecen algunos pelos alineados en su lugar (Figs. 3A, 3B). Rugosidad elitral de $M$. gomari muy difusa, casi ausente, similar a la de M. baudueri, mientras que en $M$. nanus es generalmente más acentuada y patente, si bien este carácter muestra cierta variabilidad en esta última especie. Asimismo, las genitalias masculinas de M. nanus y de M. baudueri presentan diferencias significativas entre sí y respecto a $M$. gomari: en $M$. nanus la vaina parameral es un poco más alargada, los lóbulos paramerales son rectos, nada curvados y de aspecto digitiforme en visión lateral, y el lóbulo medio muestra los dientes ventrales muy cercanos entre sí y alejados del ápice (Figs. 5A-C; Bologna, 1988: 297, figs. 96-98, 1991: 336, figs. 117d, e, f); en M. baudueri la vaina parameral es más estrecha tanto en visión dorsal como lateral y netamente más alargada que la falobase, siendo regularmente acuminada desde la base hasta el ápice en visión dorsal, sin mostrar la marcada acuminación del tercio distal de $M$. gomari, los lóbulos paramerales apenas son curvados y terminan en punta roma en visión lateral, de igual forma, los dientes ventrales del lóbulo medio están más próximos entre sí y el distal un poco más distante del ápice (Figs. 4D-F; Bologna, 1988: 297, figs. 107-109, 1991: 336, figs. 117j, k, 1).

Kocher (1956) atribuye a $M$. nanus una distribución relativamente amplia en Marruecos, donde ocuparía las regiones occidental y central del país, entre Tánger [Beni-Msuar, de donde Escalera (1914) la cita como M. baudueri], la costa atlántica y los Atlas, alcanzando los $1800 \mathrm{~m}$ de altitud en montaña (Ain-Leuh, Adassil). Bologna (1988) sólo registra una localidad marroquí, Guisser $(130 \mathrm{~km}$ al norte de Marrakech), aunque en el mapa de distribución que presenta se observan cuatro localidades (posiblemente recoja las indicadas por Kocher, 1956). Como se expuso para M. murinus, Kocher (1956: 70, nota 3) era consciente de la confusión histórica existente entre esa especie, $M$. nanus y $M$. baudueri, por lo que es muy probable que la distribución marroquí dada por este autor para $M$. nanus se ajuste en buena parte a la realidad. Anteriormente, Escalera (1914) citó igualmente a $M$. baudueri de Marrakech (suroeste de Marruecos). El examen de los ejemplares en los que basó esta cita, colectados por el mismo M. M. de la Escalera (MNCN, véase Apéndice), confirma que se corresponden realmente con $M$. nanus. Sin embargo, la cita de M. baudueri de Beni-Msuar (territorio situado al sur de Tánger, en la zona oeste de la Península Tingitana) (Escalera, 1914: 383) corresponden efectivamente a esta especie (ejemplares en el MNCN, ver Apéndice). Recientemente, 
Bologna (2008a) incluye a Marruecos y Argelia en la distribución geográfica de $M$. baudueri, aunque sin precisar registros concretos. Los datos que se presentan en el Apéndice confirman su presencia al menos en la región de Tánger. Los ejemplares de M. baudueri marroquíes estudiados muestran un tamaño menor que los ibéricos y la pilosidad un poco más corta y menos densa sobre todo en la cabeza y tergos abdominales.

Las especies exclusivas de oriente próximo y medio de este subgrupo, M. kandaharicus y $M$. omanicus, se segregan sin dificultad de M. gomari. Así, M. kandaharicus, entre otros caracteres, presenta el pronoto mucho menos transverso, aproximadamente igual de ancho que largo, subcuadrado; la macroescultura pronotal distinta, sin surco central marcado y sin depresiones laterales longitudinales; el punteado de cabeza y pronoto muy escaso y esparcido; de igual forma, su genitalia masculina presenta los lóbulos paramerales rectos y de aspecto digitiforme (Bologna, 1988: 282-285, figs. 21, 36, 49, 63, 99-102). Meloe omanicus muestra el pronoto subtrapezoidal, apenas más ancho que largo, con los ángulos laterales bien salientes y sin depresiones laterales longitudinales, tegumento de la cabeza brillante, élitros groseramente rugosos y edeago con la vaina parameral alargada, lóbulos paramerales más anchos y rectos, con el ápice redondeado, y lóbulo medio con los dientes ventrales netamente alejados del ápice (Kaszab, 1983: 186, figs.136-137, lám. 5: fig. 59).

Por su parte, M. marianii, M. saharensis, $M$. otini y $M$. vignai conforman un complejo de especies muy afines entre sí y caracterizado en el seno del subgrupo de $M$. murinus por los siguientes rasgos (Bologna, 1990), que además los separan claramente de M. gomari: tegumento corporal brillante, cabeza mirmiciforme, antenas muy largas, pronoto de lados subparalelos y estrecho, apenas más ancho que largo, patas muy alargadas y pilosidad corporal escasa, de color castaño rojizo. Además, $M$. marianii, la especie mejor conocida de este complejo junto a $M$. vignai de Somalia, presenta una genitalia muy distinta de la nueva especie, con los lóbulos paramerales rectos y de aspecto digitiforme (Bologna, 1988: 297, figs. 110-113). Según Bologna (1990: 393; 2008b: 48), M. marianii podría ser un sinónimo de Meloe saharensis Chobaut, 1898, especie descrita de Ghardaia (región central norsahariana de Argelia) y únicamente conocida por la descripción original basada en un solo ejemplar hembra (Chobaut, 1898); en tal caso, el nombre $M$. saharensis tendría prioridad sobre el nombre dado por Kaszab (1983).
Por último, Meloe punjabensis, única especie del subgénero Eurymeloe conocida en la región biogeográfica oriental (noreste de Paquistán), se diferencia de $M$. gomari, entre otros caracteres, porque aquella presenta la pilosidad corporal más larga y de color castaño-rojiza e incluso negra, el pronoto con relieves diferentes y el punteado pronotal más fino (Kaszab, 1958).

Meloe murinus, $M$. nanus y posiblemente $M$. baudueri presentan en Marruecos una extensión de presencia (sensu UICN, 2001) en parte simpátrica respecto a $M$. gomari, si bien hasta el momento no se ha detectado coincidencia entre localidades o microsimpatría entre $M$. gomari y otros Eurymeloe del subgrupo de $M$. murinus. Sin embargo, el tipo de hábitat en el que se localiza $M$. gomari, áreas de montaña hiperhúmedas con formaciones forestales mixtas de Pinus pinaster hamiltonii var. maghrebiana, $P$. nigra mauritanica y Abies maroccana (exclusivas del Rif calizo noroccidental), contrasta con los señalados para esas otras especies de Eurymeloe: M. nanus, considerada como una especie xerófila, ligada a formaciones esteparias secas, semiáridas e incluso semidesérticas, aunque también aparece en matorrales mediterráneos, con afinidad hacia áreas yesíferas en la Península Ibérica (Bologna, 1988, 1991; García-París et al., 2006; Ruiz \& García-París, 2008); M. murinus, especie termófila, ligada a pastizales, dehesas, matorrales mediterráneos, formaciones boscosas de Quercus sp. y en el Magreb también asociada a estepas xéricas (Bologna, 1988, 1991; García-París et al., 2006); y por último, M. baudueri de autoecología prácticamente desconocida, que no obstante $\mathrm{y}$ según Bologna $(1988,1991)$ parece comportarse como una especie termófila, de baja altitud, asociada a matorrales seriales y formaciones boscosas mediterráneas.

\section{AGRADECIMIENTOS}

Nuestro agradecimiento a Fco. Javier Martínez y Lotfi Mohamed por su colaboración en los muestreos realizados en las montañas de la Gomara. A Marco A. Bologna, por compartir su conocimiento del subgénero Eurymeloe con nosotros, ofrecer sus valiosas sugerencias y permitirnos revisar su importante colección. A Álvaro Velasco por sus enriquecedores comentarios sobre geografía y sociología del Rif. A Mercedes París (conservadora del MNCN, Madrid) y Gloria Masó (conservadora del Museu de Zoologia de Barcelona) por su siempre dispuesta colaboración y amabilidad. A Pepe Fernández por su ayuda en la preparación del manuscrito final. Al Instituto de Estudios Ceutíes por su continuo apoyo. Este trabajo ha sido financiado por el proyecto CGL2007-64621 del Ministerio de Ciencia e Innovación (España). 


\section{Referencias}

Al-Houty, W., 2004. A faunistic account of beetles (Coleoptera) of the state of Kuwait. Kuwait Journal of Sciences \& Engineering, 31(1): 59-76.

AksentJeV, S. I., 1988. [A catalogue of the genus-group taxa of the beetle family Meloidae (Coleoptera).] [en ruso]. Entomologicheskoye Obozreniye, 67: 569-582. [Traducción en inglés: Entomological Review, 68(3) 1989: 11-26].

AlCOCK, J., 1983. Territoriality by hilltopping males of the great purple hairstreak, Atlides halesus (Lepidoptera, Lycaenidae): convergent evolution with a pompilid wasp. Behavioral Ecology and Sociobiology, 8: 309317.

Behnstedt, P., 2002. La frontera entre el bereber y el árabe en el Rif. Estudios de dialectología norteafricana y andalusí, 6: 7-18.

BENABID, A., 1982. Etudes phytoécologique, biogéographique et dinamique des associations et séries sylvatiques du Rif occidental (Maroc). Thèse Doct. ès-Sc. Faculté des Sciences et Techniques St. Jerôme. Université de Droit, d'Economie et des Sciences d'Aix-Marseille. iii + 199 pp.

BENABID, A., 1984. Étude phytoécologique des peuplements forestiers et préforestiers du Rif centro-occidental (Maroc). Travaux de l'Institut Scientifique, Rabat, série Botanique, 34: 1-64, 25 tab., 2 cartes.

BENABID, A., 1985. Les écosystèmes forestiers, préforestiers et presteppiques du Maroc: diversité, répartition biogéographique et problèmes posés par leur aménagement. Forêt méditerranéenne, 7(1): 53-64.

BENABID, A., 2000. Flore et écosystèmes du Maroc. Évaluation et préservation de la biodiversité. Ibis Press. Paris. 359 pp.

Benabid, A. \& Fennane, M., 1994. Connaissances sur la végétation du Maroc: Phytogéographie, phytosociologie et séries de végétation. Lazaroa, 14: 21-97.

Bologna, M. A., 1988. Note su Eurymeloe e revisione delle specie euromediterranee del gruppo rugosus (Coleoptera, Meloidae). Fragmenta Entomologica, 20(2): 233-301.

BolognA, M. A., 1990. Faunistica e zoogeografia dei Meloidae (Coleoptera) della Somalia. Biogeographia, [1988], 14: 293-401.

Bologna, M. A., 1991. Fauna d'Italia. XXVIII. Coleoptera Meloidae. Calderini. Bologna. xiv +541 pp.

Bologna, M. A., 1994a. Meloidae from Canary and other macaronesian islands (Coleoptera). Miscellània Zoològica, [1992], 16: 73-80.

BolognA, M. A., 1994b. I Meloidae della Grecia (Coleoptera). Fragmenta Entomologica, 25(Supplemento): 1-119.

Bologna, M. A., 2008a. Meloidae. En: Löbl, I. \& Smetana, A. (Eds.). Catalogue of Palaearctic Coleoptera. Vol. 5. Tenebrionoidea. Apollo Books. Stenstrup: 370-412.
Bologna, M. A., 2008b. New Acts and Comments. Meloidae. En: Löbl, I. \& Smetana, A. (Eds.). Catalogue of Palaearctic Coleoptera. Vol. 5. Tenebrionoidea. Apollo Books. Stenstrup. 45-49.

Bologna, M. A., Aloisi, G. \& Marangoni, C., 1989. Nuove osservazioni su Eurymeloe Reitter e dscrizioni di larve di I stadio (Coleoptera, Meloidae). Bulletin et Annales de la Société Royale Belge d'Entomologie, 125: 67-75.

Bologna, M. A. \& Marangoni, C., 1990. Dispersal, dispersion and phoresy in the blister beetle fauna (Coleoptera, Meloidae) of eastern Mediterranean and other islands. Atti dei Convegni "Biogeographical aspects of insularity”, Accademia Nazionale dei Lincei, 85: 345-366.

Bologna, M. A. \& Pinto, J. D., 1992. A review of Meloe (Taphromeloe), including a description of the firstinstar larva of M. (T.) erythrocnemus and comments on the classification of the tribe Meloini (Coleoptera: Meloidae). Proceedings of the Entomological Society of Washington, 94(3): 299-308.

Bologna, M. A. \& Pinto, J. D., 1995. The triungulin of two palaearctic Meloe subgenera: Lasiomeloe Reitter and Micromeloe Reitter (Coleoptera, Meloidae), with bionomic and taxonomic notes. Bolletino di Zoologia, 62: 383-393.

Bologna, M. A. \& Pinto, J. D., 1998. A review of the Afrotropical species of Meloe Linnaeus, 1758 (Coleoptera, Meloidae) with descriptions of first instar larvae, a key to species and an annotated catalogue. Tropical Zoology, 11: 19-59.

Bologna, M. A. \& Pinto, J. D., 2001. Phylogenetic studies of Meloidae (Coleoptera), with special emphasis on the evolution of phoresy. Systematic Entomology, 26: 33-72.

Bologna, M. A. \& Pinto, J. D., 2002. The Old World genera of Meloidae (Coleoptera): a key and synopsis. Journal of Natural History, 36(17): 2013-2102.

Bologna, M. A. \& Turco, F., 2007. The Meloidae (Coleoptera) of the United Arab Emirates with an updated Arabian checklist. Zootaxa, 1625: 1-33.

Borchmann, F., 1917. Pars 69. Meloidae, Cephaloidae. En: E. Schenkling (ed.). Coleopterorum Catalogus auspiciis et auxilio W. Junk. Junk. Berlin. 208 pp.

CATTS, E. P., 1979. Hilltop aggregation and mating behavior by Gasterophilus intestinalis (Diptera: Gasterophilidae). Journal of Medical Entomology, 16: 461-466.

CHARCo, J., 1999. El bosque mediterráneo en el norte de África. Biodiversidad y lucha contra la desertificación. Agencia Española de Cooperación Internacional. Madrid. 370 pp.

Chobaut, A., 1898. Description de quelques espèces et variétés nouvelles de coléoptères algériens. Revue d'Entomologie, 17: 74-88. 
Cros, A., 1939. Les Meloidae des possesions françaises de l'Afrique du Nord. Étude biogéographique. Bulletin et Annales de la Société Entomologique de Belgique, 79: 247-265.

Di Giulio, A., Bologna, M. A. \& Pinto, J. D., 2002. Larval morphology of the Meloe subgenus Mesomeloe: inferences on its phylogenetic position and a first instar larval key to the Meloe subgenera (Coleoptera, Meloidae). Italian Journal of Zoology, 69: 339-344.

El Gharbaoui, A., 1981. La terre et l'homme dans la Peninsule Tingitane. Etude sur l'homme et le milieu naturel dans le Rif occidental. Travaux de l'Institut Scientifique, série Géologie et Géographie physique, 15: 1-439, 4 lám., 8 mapas.

EsCALERA, M. M. DE LA, 1909. Especies nuevas de Meloidos del SW. de Marruecos. Boletín de la Real Sociedad Española de Historia Natural, 9: 240-249.

EscalerA, M. M. DE LA, 1914. Los Coleópteros de Marruecos. Trabajos del Museo Nacional de Ciencias Naturales, serie Zoológica, 11: 1-553.

EsCHERICH, K., 1890. Revision der behaarten MeloëArten der alten Welt. Wiener Entomologische Zeitung, 9: 87-96.

GARcíA, R., 2008. Distribución de la familia Meloidae (Coleoptera) en la Isla de La Palma. Revista de Estudios Generales de la Isla de La Palma, [2007], 3: 363-380.

GarcíA-París, M. \& PARrA-OleA, G., 2003. Una agrupación reproductiva de tipo "hilltopping" en Meloe strigulosus Mannerheim (Coleoptera: Meloidae). Boletín de la Sociedad Entomológica Aragonesa, 32: 81-82.

García-París, M., Trotta-Moreu, N. \& CApote, L., 2006. Estado de conocimiento actual y problemas de conservación de los Meloidae (Coleoptera) de la Comunidad de Madrid. Graellsia, 62(número extraordinario): 333-370.

HAJJARABI, F., 1991. Sauver la forêt ou sauver les femmes: corvée de bois chez les gumara. En: Zouggari, A. \& Vignet-Zunz, J. (Coords.). Jbala, historie et société. Etudes sur le Maroc du Nord-Ouest. Editions du CNRS/Wallada. Paris-Casablanca: 373394.

KASZAB, Z., 1958. Die Meloiden Afghanistan (Coleoptera). Acta zoologica Academiae scientiarum Hungaricae, 3: 245-312.

KASZAB, Z., 1983. Insects of Saudi Arabia. Coleoptera: Fam. Meloidae. A synopsis of the Arabian Meloidae. En: Wittmer, W. \& Büttiker, W. (Eds.). Fauna of Saudi Arabia. Vol. 5. Pro Entomologia c/o Natural History Museum, Basle and Meterorology and Environmental Protection Administration, Jeddah. Basle (Switzerland): 144-204.

KochER, L., 1938. Localisations nouvelles ou intéressantes de coléoptères marocains. Bulletin de la Société de Sciences Naturelles du Maroc, 18: 77-118.
Kocher, L., 1956. Catalogue commenté des Coléoptères du Maroc. V. Héteromères (Tenebrionides excepts). Travaux de l'Institut Scientifique Chérifien (série Zoologie), 10: 1-107.

Kocher, L., 1964. Catalogue commenté des Coléoptères du Maroc. X. Addenda et Corrigenda. Tables. Travaux de l'Institut Scientifique Chérifien (série Zoologie), 30: 1-200.

LÜCKMANN, J. \& Scharf, S., 2004. Description of the first instar larvae of three species of Meloe with a key to the triungulins of Central European species of this genus (Coleoptera: Meloidae). European Journal of Entomology, 101: 313-322.

Machado, A. \& Oromí, P., 2000. Elenco de los Coleópteros de las Islas Canarias. Instituto de Estudios Canarios, Monografía 70. La Laguna. 306 pp.

Molina VÁzQuez, F., (Coord.), 2006. Reserva de la Biosfera Intercontinental del Mediterráneo Andalucía (España)-Marruecos. Junta de Andalucía, Consejería de Medio Ambiente. Sevilla. 209 pp.

Nogué, J. \& Villanova, J. L., 1999. La zona norte del protectorado español en Marruecos. El marco geográfico. En: Nogué, J. \& Villanova, J.L. (Eds.). España en Marruecos (1912-1956). Discursos geográficos e intervención territorial. Milenio. Lleida: 101-141.

Pardo Alcaide, A., 1951. Estudios sobre Meloidae. III. Una nueva especie de Meloe de la isla de Tenerife y comentarios sobre algunos meloideos de la citada isla. Eos, Revista Española de Entomología, 25: 249255.

Pérez-Moreno, I., SAn Martín, A.F. \& Recalde IRURZUN, J. I., 2003. Aportaciones corológicas y faunísticas sobre meloidos ibéricos (Coleoptera: Meloidae). Boletín de la Sociedad Entomológica Aragonesa, 33: 195-217.

Peyerimhoff, P. DE, 1949. Études et descriptions des coléoptères marocains II. Bulletin de la Société de Sciences Naturelles et Physiques du Maroc, [194547], 25-27: 248-308.

Pinto, J. D. \& Selander, R. B., 1970. The bionomics of blister beetles of the genus Meloe and a classification of the New World species. Illinois Biological Monographs, 42: 1-222.

Quézel, P., Barbéro, M., Benabid, A., Loisel, R. \& Rivas-Martínez, S., 1988. Contribution à l'etude des groupements préforestiers et des matorrals rifains. Ecologia mediterranea, 14(1/2): 77-122.

Rivas-MartínEZ, S., 1987. Memoria del mapa de las series de vegetación de España 1:400.000. ICONA. Madrid. 268 pp.

Ruiz, J. L., Ávila, J. M. \& SAlado, R., 1993. Confirmación de la presencia de Meloe (Eurymeloe) baudueri Grenier, 1863 en la Península Ibérica (Coleoptera, Meloidae). Boletín de la Asociación española de Entomología, 17(1): 203. 
Ruiz, J. L. \& GARcíA-PARÍs, M., 2008. Meloe (Eurymeloe) nanus Lucas, 1849. En: Barea-Azcón, J.M., Ballesteros-Duperón, E. \& Moreno, D., (Coords.). Libro Rojo de los Invertebrados de Andalucía. Tomo III. Consejería de Medio Ambiente, Junta de Andalucía. Sevilla: 1030-1037.

SAnz de GALdEAno, C., 1997. La zona interna béticorifeña. Colección Monográfica Tierras del Sur, Universidad de Granada. Granada. 316 pp.

SElander, R. B., 1985. Spastomeloe, a new genus of Meloini from Peru (Coleoptera: Meloidae). Journal of the Kansas Entomological Society, 58: 668-685.

SElAnder, R. B., 1989. The triungulin larva of Meloe cicatricosus Leach (Col., Meloidae). Deutsche Entomologische Zeitschrift (N.F.), 36: 401-407.

SElander, R. B., 1991. On the Nomenclature and Classification of the Meloidae (Coleoptera). Insecta Mundi, 5(2): 65-94.

SHIELDS, O., 1967. Hilltopping. Journal of Research on the Lepidoptera, 6: 69-178.
UICN (Unión Internacional para la Conservación de la Naturaleza), 2001. Categorías y Criterios de las Listas Rojas de la UICN: Versión 3.1. Comisión de Supervivencia de Especies de la UICN, UICN. Gland \& Cambridge. ii +33 pp.

WILDI, W., 1979. Evolution de la plate-forme carbonatée de type austro-alpin de la Dorsale calcaire (Rif interne, Maroc septentrional) au Mésozoïque. Bulletin de la Société Géologique de France, (7), 21(1): 49-56.
Recibido, 3-IV-2009

Aceptado, 28-X-2009 Publicado, 31-XII-2009 
Apéndice.- Material examinado del subgénero Eurymeloe. Las siglas utilizadas son las siguientes: MNCN: Museo Nacional de Ciencias Naturales (Madrid, España); MZB: Museu de Zoologia (Barcelona, España); MAB: Colección Marco Bologna (Universidad Roma Tre, Roma, Italia); JLR: Colección de José L. Ruiz (Ceuta, España).

Appendix. - Material examined of subgenus Eurymeloe. The acronyms used are as follows: MNCN: Museo Nacional de Ciencias Naturales (Madrid, Spain); MZB: Museu de Zoologia (Barcelona, Spain); MAB: Marco Bologna's collection (Roma Tre University, Roma, Italy); JLR: José L. Ruiz's collection (Ceuta, Spain).

\section{Meloe (Eurymeloe) baudueri Grenier, 1863}

MARRUECOS: 2 ejemplares: "Tánger / M. Escalera" (MNCN); 7 ejemplares: "Beni-Msuar” (MNCN).

ESPAÑA: ALMERÍA: 1 ejemplar: “Covatillas” (MNCN); SEVILLA: 1 ejemplar: "Sevilla", "Meloe (Eurymeloe) baudueri Grenier / M.A. Bologna det. 1992" (MNCN); MADRID: 1 ejemplar: "Montarco / 15-4", "Meloe (Eurymeloe) nanus Lucas ? / M.A. Bologna det. 1992" (MNCN): este ejemplar fue citado como Meloe (Eurymeloe) nanus en García-París et al. (2006), registro que aquí atribuimos a $M$. (E.) baudueri.

\section{Meloe (Eurymeloe) flavicomus Wollaston, 1854}

ESPAÑA: ISLAS CANARIAS: 3 ejemplares: "Canarias / La Gomera / 1-64 / J. Gil leg." (MZB); 1 ejemplar: "Bosque del Cedro / Gomera, 900-1.100 m. / IV-1935, Bolívar-Bonet" $(\mathrm{MNCN})$

PORTUGAL: MADEIRA: 1 ejemplar: "Meloe flavicomus Woll. / Madeira / Paiva", "Col. del Sr. Pérez-Arcas" $(\mathrm{MNCN})$.

Meloe (Eurymeloe) murinus Brandt et Erichson, 1832

ESPAÑA: ALBACETE: 1 ejemplar: "Alcaráz", "Meloe (Eurymeloe) murinus Br. et Er. / M. G. París \& L. Capote det. 1992" (MNCN); 1 ejemplar: "Nerpio", "Meloe (Eurymeloe) murinus Br. et Er. / M. G. París \& L. Capote det. 1992" (MNCN). GRANADA: 1 ejemplar: "umg. Granada / I-1977 / Krätschmer leg." (MAB). MADRID: 1 ejemplar: "Alcalá de H.[Henares]", "Meloe (Eurymeloe) murinus Br. et Er. / M.A. Bologna det. 1992" (MNCN); 1 ejemplar: "Meloe murinus Brandt / Madrid", "Meloe (Eurymeloe) murinus Br. et Er. / M. G. París \& L. Capote det. 1992" (MNCN); 1 ejemplar: Colmenar Viejo / 9-XI1984 / F.A. Montes (MNCN); 1 ejemplar: $5 \mathrm{~km}$ al E de Colmenar Viejo / 30-X-2005 / M. García París leg. (MNCN, col EtOH).

Meloe (Eurymeloe) nanus Lucas, 1847

MARRUECOS: 8 ejemplares: "Marruecos / Marraquesh / I1907 - Escalera" (1 ejemplar etiquetado "Meloe (Eurymeloe) nanus Lucas / M.A. Bologna det. 1992"; 1 ejemplar etiqueta- do "M. Baudueri Gren." "Meloe (Eurymeloe) nanus Lucas / M.A. Bologna det. 1992”)(MNCN); 1 ejemplar: “Azrou / 9-41923" (MNCN); 1 ejemplar: "Marruecos / Gran Atlas/ Ait M'Hamed / 31-XII-68" (MZB); 1 ejemplar: "Izmoren (Bocoya) / Melilla-Marruecos / Pardo Alcaide/ III-1934" (MZB).

ESPAÑA: ALICANTE: 1 ejemplar: "Daya", "Baudueri Gren.” (MNCN). ALMERÍA: 1 ejemplar: "w Sorbas / XII-1977 / Krätschmer leg." (MAB). CUENCA: 1 ejemplar: "Uclés / Pantel", "Meloe (Eurymeloe) nanus Lucas / M.A. Bologna det. 1992"(MNCN); 2 ejemplares: "Uclés", "Meloe (Eurymeloe) nanus Lucas / M.A. Bologna det. 1992"(MNCN);1 ejemplar: "Uclés / Meloe Baudueri Grenier" (MNCN); 1 ejemplar: "M. baudueri / Greni. / Uclés" (MNCN). GRANADA: 1 ejemplar: "Ladihonda / Guadix (Granada) / 30SVG9730 / 17-I-1993, bajo piedra / F.S. Piñero leg." (JLR). JAÉN: 1 ejemplar: "Centenillo / Sierra Morena / Juan Cabre", "Meloe (Eurymeloe) nanus Lucas / M.A. Bologna det. 1992")(MNCN); 1 ejemplar: "Meloe / Paz / L.[Lomas] de Úbeda", "Col. del Sr. Pérez Arcas", "Meloe (Eurymeloe) nanus Lucas / M.A. Bologna det. 1992" (MNCN). MADRID: 1 ejemplar: "Meloe L. murinus B. et E. / (Aranjuez)", "Col. del Sr. Pérez Arcas", "Meloe (Eurymeloe) nanus Lucas / M.A. Bologna det. 1992" (MNCN); 1 ejemplar: $3 \mathrm{~km}$ al S de Tielmes / 3-XI-2000 / J. L. Ruiz \& M. García París leg. (MNCN, col EtOH). MURCIA: 1 ejemplar: "Jumilla" (MNCN).

Meloe (Eurymeloe) pallidicolor Escalera, 1909

MARRUECOS: 5 ejemplares (sintipo): "Marruecos / Mogador /XII-1906-Escalera" (etiqueta blanca, impresa)(MNCN); 1 ejemplar (sintipo): "Marruecos / Mogador /XII-1905 Escalera" (etiqueta blanca, impresa), "pallidicolor Esc. tipos" (etiqueta blanca, manuscrita) (MNCN); 1 ejemplar (sintipo): "Marruecos / Mogador /XII-1905-Escalera" (etiqueta blanca, impresa), "M. pallidicolor Esc." (etiqueta blanca, manuscrita) (MNCN); 2 ejemplares (sintipo): "Marruecos / Mogador /VII-1905-Escalera" (etiqueta blanca, impresa)(MNCN); 1 ejemplar (sintipo): "Marruecos / Mogador /VII-1905-Escalera" (etiqueta blanca, impresa), "Meloe pallidicolor Esc. Pardo Alcaide det., 1952" (etiqueta blanca, parte impresa, parte manuscrita) (MNCN); los 10 ejemplares portando además las siguientes etiquetas: "Sintipo" (etiqueta roja, impresa), "MNCN / Cat. Tipos n 1912 " (etiqueta roja, impresa). 